\title{
Second-Order Expansions for a Class of Quasidifferentiable Functions
}

Z.Q. Xia

Thao Chunjun

July 1987

WP-87-068

Working Papers are interim reports on work of the International Institute for Applied Systems Analysis and have received only limited review. Views or opinions expressed herein do not necessarily represent those of the Institute or of its National Member Organizations.

INTERNATIONAL INSTITUTE FOR APPLIED SYSTEMS ANALYSIS

A-2361 Laxenburg, Austria 


\section{Foreword}

Some results concerning second order expansions for quasidifferentiable functions in the sense of Demyanov and Rubinov whose gradients are quasidifferentiable are represented in this paper. They are similar to those given by HiriartUrruty, Strodiot and Nguyen (1984).

Alexander B. Kurzhanski

Chairman

System and Decision Sciences Program 


\title{
Second-Order Fxpansions for a Class of Quasidifferentiable Functions
}

\author{
Z.Q. Xia and Zhao Chunjun
}

\section{INTRODUCTION - SOME RESULTS IN [5]}

Some results concerning the second-order expansion for $C^{1.1}$ functions which are continuously differentiable and whose gradients are locally Lipschitzian. Several results given in [5] are listed as follows:

Definition 1.1. [Def. 2.1]. Let $f \in C^{1.1}(S)$ and let $x \in S$, where $S$ is an open set in $\mathbf{R}^{n}$. The generalized Hessian matrix of $f$ at $x$ is defined by

$$
\operatorname{co}\left\{M \mid \exists\left\{x_{i}\right\}_{1}^{\infty} \rightarrow x \text { such that } H_{f}\left(x_{i}\right) \text { exist and } M=\lim _{i \rightarrow \infty} H_{f}\left(x_{i}\right)\right\}
$$

where $H_{f}\left(x_{i}\right)$ denotes the Hessian matrix of $f$ at $x$. By $\partial^{2} f(x)$ one denotes the generalized matrix of $f$ at $x$.

The generalized Hessian matrix of $f$ at $x$ is a nonempty compact convex set of symmetric matrices. The set-valued mapping $x \rightarrow \partial^{2} f(x)$ is locally bounded, i.e.,

$$
\sup \left\{\|M\| \mid M \in \partial^{2} f(V)\right\} \leq K \text {. }
$$

where $V$ is a neighborhood of $x$ and $K$ is a positive constant. The mapping $\partial^{2} f(\cdot)$ is an upper semicontinuous (closed) set-valued mapping in the sense of sequences.

Theorem 1.2. [Th. 2.1. and Th. 2.2.]. Let $f \in C^{1.1}(S), F=\left(f_{1}, \ldots, f_{m}\right)^{T}$ where $f_{1} \in C^{1.1}(S), \varphi: S^{\prime} \subset \mathrm{R}^{m} \rightarrow \mathrm{R}$ where $S^{\prime}$ is an open set and $F(S) \subset S^{\prime}$. Then

$$
\begin{aligned}
f^{00}(x ; u, v)= & \left.\lim _{\substack { x, i x \\
\begin{subarray}{c}{i \neq 0 \\
\text { sup }{ x , i x \\
\begin{subarray} { c } { i \neq 0 \\
\text { sup } } }\end{subarray}} \frac{1}{\lambda}\left[\left\langle\nabla f\left(x^{\prime}+\lambda u\right), v\right\rangle-\nabla f(x), v\right\rangle\right], x \in S ; \\
(\varphi \cdot F)^{00}(x ; u, v) \leq & \sum_{i=1}^{m} \frac{\partial \varphi}{\partial y_{t}}(F(x)) f_{i}^{00}(x ; u, v) \\
& +\varphi^{00}(F(x) ; J F(x) u, J F(x) v)
\end{aligned}
$$


and the equality holds whenever either $f_{i} \in C^{2}(S)$ for all $i$, or $\varphi \in C^{2}\left(S^{\prime}\right)$ and $m=1$;

$$
\begin{aligned}
\partial^{2}(\varphi \cdot F)(x) u & \subset \sum_{i=1}^{m} \frac{\partial \varphi}{\partial y_{i}}(F(x)) \partial^{2} f_{i}(x) u \\
& +J^{T} F(x) \theta^{2} \varphi(F(x)) J F(x) u
\end{aligned}
$$

for all $u \in \mathbf{R}^{n}$.

Theorem 1.3. [Th. 2.3]. Let $f \in C^{1.1}$ and $x+\lambda d \in S$ where $\lambda>0$ and $x \in S$. Then there exists a $C \in(0, \lambda)$ such that

$$
f(x+\lambda \alpha)=f(x)+\lambda\langle\nabla f(x), d\rangle+\frac{\lambda^{2}}{2}\left\langle M_{c} \alpha, \alpha\right\rangle .
$$

where $M_{c} \in \partial^{2} f(x+c d)$.

Theorean 1.4. [Th. 3.1]. Let $x^{*}$ be a minimum point for

$$
\min f(x) \text { over all } x \text {. }
$$

Then for each $\alpha \in \mathbb{R}^{n}$ there exists a matrix $A \in \partial^{2} f\left(x^{*}\right)$ such that

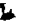

$$
\langle A \alpha, \alpha\rangle \geq 0
$$

In this paper some similar results for quasidifferentiable functions will be derived.

\section{QUASI-JACOBLAN MATRICES AND SECOND-ORDER QUASIDIFFERENTIALS}

Suppose $H$ is a mapping, a vector-valued function,

$$
H \cdot \mathbf{R}^{\boldsymbol{n}} \rightarrow \mathbf{R}^{\mathbf{m}}
$$

defined by

$$
x \mapsto H(x)=\left(h_{1}(x), h_{2}(x), \ldots, h_{m}(x)\right)^{T},
$$

where $h_{i}(x), i=1, \ldots, m$, are quasidifferentiable functions defined in $R^{n}$. Define

$$
J_{D} H:=\left[J_{D} H, \bar{J}_{D} H\right],
$$

where 


$$
\begin{aligned}
& \underline{J}_{D} H:=\left(\prod_{i=1}^{M} \underline{\partial} h_{i}\right)^{T}, \\
& \bar{J}_{D} H:=\left(\prod_{i=1}^{m} \bar{\partial} h_{i}\right)^{T} .
\end{aligned}
$$

Proposition 2.1. Suppose $H$ is a quasidifferentiable mapping $\left(\mathbf{R}^{n} \rightarrow \mathbf{R}^{m}\right)$, [3],

[4]. Then $J_{D} H(x)$ is one of quasidifferentials of the mapping $H$ at $x \in \mathbf{R}^{n}$.

Proof. In a finite dimensional space $\mathbf{R}^{m}$ we have

$$
\begin{aligned}
H^{\prime}(x ; \alpha) i & =\lim _{\lambda \downarrow 0} \frac{1}{\lambda}[H(x+\lambda \alpha)-H(x)] \\
& =\lim _{\lambda \downarrow 0} \frac{1}{\lambda}\left[h_{1}(x+\lambda \alpha)-h_{1}(x)\right], \cdots . \\
& \left.-\lim _{\lambda \downarrow 0} \frac{1}{\lambda}\left[h_{m}(x+\lambda d)-h_{m}(x)\right]\right)^{T} .
\end{aligned}
$$

Thus

$$
\max _{V \in Q H(x)}\langle V, \alpha\rangle+\min _{W \in \hat{\theta} H(x)}\langle W, \alpha\rangle=\max _{V \in \mathcal{I}_{D} H(x)}\langle V, \alpha\rangle+\min _{W \in \bar{J}_{D H} H(x)}\langle W, \alpha\rangle
$$

i.e., for any $\alpha \in \mathbf{R}^{n}$ one has

$$
\underline{\theta} H(x)-\bar{J}_{D} H(x)=J_{D} H(x)-\bar{\partial} H(x) .
$$

Since $\underline{J}_{D} H(x), \bar{J}_{D} H(x)$ are nonempty compact convex sets, it follows from the definition of quasidifferentiable functions that

$$
J_{D} H(x)=\left[J_{D} H(x), \bar{J}_{D} H(x)\right]
$$

is a quasidifferential of $H$ at $x$.

It is easy to be seen that

$$
D H(x)=\left\{\begin{array}{ll}
J_{D} H(x), & \text { if } m>1 \\
J_{D}^{T} H(x), & \text { if } m=1
\end{array},\right.
$$

where

$$
J_{D}^{T} H(x):=\left[J_{D}^{T} H(x), \bar{J}_{D}^{T} H(x)\right]
$$

[7]. 
Remark. In the case where $H$ is Lipschitzian the relationship, generally speaking,

$$
\begin{aligned}
\prod_{i=1}^{m} \partial_{c} h_{i}(x) & =\theta_{c} H(x) \\
& =J_{c} H(x)
\end{aligned}
$$

is not true, but in the case where $H(x)$ is quasidifferentiable the pair

$$
\left[\stackrel{m}{X}_{i=1}^{m} \underline{\partial} h_{i}(x),{\underset{i=1}{X}}_{\bar{\theta}}^{m} h_{i}(x)\right]
$$

is really a quasidifferential of $H$ at $x \in \mathbf{R}^{n}$, where $\partial_{c}$ denotes the Clarke's generalized matrix or generalized Jacobian matrix, [1], [2].

Definition 2.2. $J_{D} H(x)$ is called a quasi-Jacobian matrix of the quasidifferentiable mapping $H . \underline{J}_{D} H(x)$ is called sub-Jacobian matrix, and $\bar{J}_{D} H(x)$ is called super-Jacobian matrix, [7].

Definition 2.3. Suppose $f$ is a differentiable function defined in $\mathbf{R}^{\boldsymbol{n}}$ and its gradient is a quasidifferentiable mapping, vector-valued function. A quasiJacobian matrix of its gradient $J_{D} \nabla f(x)$ is called a second-order quasidifferential of $f$ at $x \in \mathbf{R}^{n}$. The sub-Jacobian matrix is called a second-order subquasidifferential. The super-Jacobian matrix is called a second-order superquasidifferential. They are denoted by $D^{2} f(x), \underline{\theta}^{2} f(x)$ and $\bar{\partial}^{2} f(x)$, respectively. i.e.,

$$
\begin{gathered}
D^{2} f(x)=\left[\underline{J}_{D} \nabla f(x), \bar{J}_{D} \nabla f(x)\right], \\
\partial^{2} f(x)=\Sigma_{D} \nabla f(x), \bar{\theta}^{2} f(x)=\bar{J}_{D} \nabla f(x) .
\end{gathered}
$$

Theorem 2.4. Suppose $f$ is a differentiable function defined in $\mathbf{R}^{n}$ and its gradient is quasidifferentiable. Then 


$$
\begin{aligned}
& \frac{\partial^{2} f(x)}{\partial d_{1} \partial d_{2}}=f^{\prime \prime}\left(x ; \alpha_{1}, d_{2}\right) \\
& =\max _{\substack{V \in Q^{2} f(x) \\
V \in \bar{C}_{f}(x)}}\left(\alpha_{1}^{+T_{V}}-\alpha_{1}^{-T_{W}}\right) \alpha_{2} \\
& +\min _{\substack{V \in g^{2} f(x) \\
V \in d_{f}(x)}}\left(\alpha_{1}{ }^{+T_{W}}-\alpha_{1}{ }^{-T_{V}}\right) \alpha_{2} \\
& =\max \left(\alpha_{1}^{+T} \partial^{2} f(x)-\alpha_{1}{ }^{-T} \bar{\partial}^{2} f(x)\right) \alpha_{2} \\
& +\min \left(\alpha_{1}^{+T} \bar{\partial}^{2} f(x)-\alpha_{1}{ }^{-T} \underline{\partial}^{2} f(x)\right) \alpha_{2} .
\end{aligned}
$$

where $\alpha_{1}=\alpha_{1}^{+}-\alpha_{1}^{-}, \alpha_{1}^{+}$and $d_{1}^{-}$are defined, respectively, by

$$
\alpha_{1}^{+}=\left(\alpha_{1 i}^{+}\right)_{n \times 1}, \quad \alpha_{1 i}^{+}= \begin{cases}\alpha_{1 i}, & \alpha_{1 i} \geq 0, \\ 0, & \alpha_{1 i}<0,\end{cases}
$$

and

$$
\alpha_{1}^{-}=\left(\alpha_{1 i}^{-}\right)_{n \times 1}, d_{1 i}= \begin{cases}0, & d_{1 i} \geq 0 \\ -d_{1 i}, & \alpha_{1 i}<0 .\end{cases}
$$

Proof. Since

$$
\begin{aligned}
\frac{\partial^{2} f(x)}{\partial \alpha_{1} \partial \alpha_{2}} & =f^{\prime \prime}\left(x ; \alpha_{1}, \alpha_{2}\right) \\
& =\lim _{\lambda \downarrow 0} \frac{1}{\lambda}\left[\left\langle\nabla f\left(x+\lambda \alpha_{2}\right), \alpha_{1}\right\rangle-\left\langle\nabla f(x), \alpha_{1}\right\rangle\right] \\
& =\lim _{\lambda+0}\left\langle\frac{1}{\lambda}\left(\nabla f\left(x+\lambda \alpha_{2}\right)-\nabla f(x)\right), \alpha_{1}\right\rangle \\
& =\lim _{\lambda+0_{i}} \sum_{i=1}^{n} \frac{1}{\lambda}\left\langle\nabla f\left(x+\lambda \alpha_{2}\right)-\nabla f(x)\right)_{1} \alpha_{1 i} \\
& =\sum_{i=1}^{n} d_{1 i}\left[\max _{v_{i} \in \theta(\nabla f(x))_{i}}\left\langle v_{i}, d_{2}\right\rangle+\min _{\left.w_{i} \in \bar{\theta}(\nabla f(x))_{i}\left\langle w_{i}, d_{2}\right\rangle\right],}\right.
\end{aligned}
$$

one has 


$$
\begin{aligned}
f^{\prime \prime}\left(x ; \alpha_{1}, \alpha_{2}\right) & =\left\{\sum\left[\alpha_{1 i}^{+} \max _{v_{i} \in Q(\nabla f(x))_{i}}\left\langle v_{i}, \alpha_{2}\right\rangle+\alpha_{1 i}^{+} \min _{w_{i} \in \bar{\theta}(\nabla f(x))_{i}}\left\langle w_{i}, \alpha_{2}\right\rangle\right]\right. \\
& \left.+\left[-\alpha_{1 i} \max _{v_{i} \in Q(\nabla f(x))_{i}}\left\langle v_{i}, \alpha_{2}\right\rangle-\alpha_{1 i} \bar{w}_{w_{i} \in \bar{\theta}(\nabla f(x))_{i}}\left\langle w_{i}, \alpha_{2}\right\rangle\right]\right\} .
\end{aligned}
$$

Therefore,

$$
\begin{aligned}
& f^{\prime \prime}\left(x ; \alpha_{1}, d_{2}\right)=\max _{\substack{V \in g^{Q} f(x) \\
v \alpha_{d}^{-} d_{f}(x)}}\left\langle V^{T} d_{1}^{+}-W^{T} \alpha_{1}^{-}, \alpha_{2}\right\rangle \\
& +\min _{\substack{V \in g^{2} f(x) \\
v \in \bar{d}_{f}(x)}}\left\langle-V^{T} \alpha_{1}^{-}+W^{T} \alpha_{1}^{+}, \alpha_{2}\right\rangle .
\end{aligned}
$$

The (2.1) can be obtained immediately from (2.2).

By $\diamond$ we denote an operation as follows:

$$
\alpha \diamond[A, B]:=\left[\alpha^{T} \diamond_{1}[A, B], \alpha^{T} \diamond_{2}[A, B]\right]
$$

where

$$
\alpha^{T} \diamond_{1}[A, B]:=\alpha^{+T} A-\alpha^{-T} B
$$

and

$$
\alpha^{T} \diamond_{2}[A, B]:=\alpha^{+T} B-\alpha^{-T} A
$$

here $d$ is a vector in $R^{n}, A$ and $B$ are matrices or sets of matrices. Using the notations given above, we may write $f^{\prime \prime}\left(x ; \alpha_{1}, \alpha_{2}\right)$ as follows:

$$
f^{\prime \prime}\left(x ; \alpha_{1}, \alpha_{2}\right)=\max \alpha_{1}^{T} \diamond_{1} D^{2} f(x) \alpha_{2}+\min \alpha_{1}^{T} \diamond_{2} D^{2} f(x) \alpha_{2} .
$$

Note that generally speaking,

$$
\alpha_{1}^{T} \diamond_{i} D^{2} f(x) \alpha_{2} \neq \alpha_{2}^{T} \diamond_{i} D^{2} f(x) \alpha_{1}, \quad i=1,2
$$

Proposition 2.5. The following relation holds

$$
\alpha_{1}^{T} \diamond_{1} D^{2} f(x) \alpha_{2}+\alpha_{1}^{T} \diamond_{2} D^{2} f(x) \alpha_{2}=\alpha_{1}^{T}\left(\partial^{2} f(x)+\bar{\partial}^{2} f(x)\right) \alpha_{2}^{T} .
$$

Proposition 2.6. Suppose $f_{1}$ and $f_{2}$ are differentiable functions defined in $\mathbf{R}^{n}$, and their gradients are quasidifferentiable. Then

$$
D^{2}\left(\alpha f_{1}+\beta f_{2}\right)=\alpha D^{2} f_{1}+\beta D^{2} f_{2} .
$$

where $\alpha$ and $\beta$ are any scalars. And 


$$
\begin{aligned}
D^{2}\left(f_{1} \cdot \rho_{2}\right) & =\rho_{1} \cdot J_{D} \nabla \rho_{2}+\rho_{2} \cdot J_{D} \nabla \rho_{1} \\
& +\nabla \rho_{1} \otimes J_{D} \rho_{2}+\nabla \rho_{2} \otimes J_{D} \rho_{1} \\
& =\rho_{1} D^{2} \rho_{2}+\rho_{2} D^{2} \rho_{1}+\nabla \rho_{1} \otimes D^{T} \rho_{2}+\nabla f_{2} \otimes D^{T} f_{1}
\end{aligned}
$$

where the operation $\otimes$ means that

$$
\nabla f_{1} \otimes J_{D} f_{2}=\left[\begin{array}{c}
\vdots \\
\left(\nabla f_{1}\right)_{1} J_{D} f_{2} \\
\vdots
\end{array}\right]
$$

Proof. The proof of (2) will be given below. Since

$$
\begin{aligned}
D^{2}\left(f_{1} \cdot f_{2}\right) & =D\left(f_{1} \nabla f_{2}+f_{2} \nabla f_{1}\right) \\
& =J_{D}\left(f_{1} \nabla f_{2}\right)+J_{D}\left(f_{2} \nabla f_{1}\right),
\end{aligned}
$$

it is enough to prove that one of the terms in the last expression given above, say, the first term $J_{D}\left(f_{1} \nabla f_{2}\right)$ can be obtained. Since

$$
J_{D}\left(f_{1} \nabla f_{2}\right)=J_{D}\left[\begin{array}{c}
\vdots \\
\rho_{1}\left(\nabla f_{2}\right)_{i} \\
\vdots
\end{array}\right],
$$

one has

$$
\underline{J}_{D}\left(f_{1} \nabla f_{2}\right)=\prod_{i=1}^{n} \partial\left(f_{1}\left(\nabla f_{2}\right)_{i}\right), \quad \bar{J}_{D}\left(f_{1} \nabla f_{2}\right)=\prod_{i=1}^{n} \bar{\partial}\left(f_{1}\left(\nabla f_{2}\right)_{i}\right)
$$

Consider the $J_{D}^{T}\left(f_{1}\left(\nabla f_{2}\right)_{i}\right)$. According to the definition of $T$ we have

$$
\begin{aligned}
J_{D}^{T}\left(f_{1}\left(\nabla f_{2}\right)_{i}\right) & =D\left(\rho_{1}\left(\nabla f_{2}\right) i\right) \\
& =\left(\nabla f_{2}\right)_{i} D f_{1}+f_{1} D\left(\nabla f_{2}\right)_{i} \\
& =\left(\nabla f_{2}\right)_{i} J_{D}^{T} \rho_{1}+\rho_{1} J_{D}^{T}\left(\nabla \rho_{2}\right)_{i} .
\end{aligned}
$$

From the above one has

$$
J_{D}\left(f_{1}\left(\nabla f_{2}\right)_{i}\right)=\left(\nabla f_{2}\right)_{i} J_{D} f_{1}+f_{1} J_{D}\left(\nabla f_{2}\right)_{i}
$$

Similarly, we can get

$$
J_{D}\left(f_{2}\left(\nabla f_{1}\right)_{i}\right)=\left(\nabla \rho_{1}\right)_{i} J_{D} f_{2}+f_{2} J_{D}\left(\nabla \rho_{1}\right)_{i}
$$

The proof of (2) can be completed immediately from (2.3) and (2.4). 


\section{SECOND-ORDER EXPANSION}

To begin with, we give the following lemma, Cauchy's Mean Value Theorem, in order to deduce second-order expansions.

Lemma 3.1. (Cauchy's Mean Value Theorem). Suppose $f, g: \mathbf{R}^{n} \rightarrow \mathbf{R}$ are quasidifferentiable and for an interval $[x, y] \subset \mathbf{R}^{n}$

$$
0 \notin\langle\underline{\partial g} g(\xi)+\bar{\partial} g(\xi), y-x>, \xi \in(x, y) .
$$

Then there exists an $\eta \in(x, y)$ such that

$$
\frac{f(y)-f(x)}{g(y)-g(x)} \in \frac{\langle\partial f(\eta)+\bar{\partial} f(\eta), y-x\rangle}{\langle\partial g(\eta)+\bar{\partial} g(\eta), y-x\rangle}
$$

Proof. The proof can be made along the lines of a process used in the proof of Cauchy's Mean Value Theorem in the elementary calculus. According to [7, Th. 2], the fact $g(x) \neq g(y)$ is true. Thus we can construct an auxiliary function

$$
F(\xi)=f(\xi)-\frac{f(y)-f(x)}{g(y)-g(x)} g(\xi) .
$$

Without loss of generality, assume that

$$
\frac{f(y)-f(x)}{g(y)-g(x)} \leq 0
$$

It follows from [7. Th. 2] that there exists an $\eta \in(x, y)$ such that

$$
F(y)-F(x) \in\langle\underline{\partial} F(\eta)+\bar{\partial} F(\eta), y-x\rangle \text {. }
$$

We now have to find $D F(\eta)$. From the rules in quasidifferential calculus one has

$$
\begin{aligned}
D F(\eta) & =D f(\eta)-\frac{f(y)-f(x)}{g(y)-g(x)} D g(\eta) \\
& =\left[\partial f(\eta)-\frac{f(y)-f(x)}{g(y)-g(x)} \partial g(\eta),\right. \\
& \left.\bar{\partial} f(\eta)-\frac{f(y)-f(x)}{g(y)-g(x)} \bar{\partial} g(\eta)\right] .
\end{aligned}
$$

[3], [4]. It is easy to learn by directly calculating $F$ that

$$
F(y)-F(x)=0
$$

Thus we have

$$
0 \in\left\langle\partial f(\eta)+\bar{\partial} f(\eta)-\frac{f(y)-f(x)}{g(y)-g(x)}(\theta g(\eta)+\bar{\partial} g(\eta)), y-x\right\rangle .
$$


The proof is completed immediately from the hypothesis of this theorem (3.1).

Remark. Let $A$ and $B$ be subsets in $\mathbf{R}$. The quotient set $A / B=\frac{A}{B}$ is defined as

$$
A / B=\left\{\frac{a}{b} \mid a \in A, b \in B\right\}
$$

where $a / b$ can be treated as a usual quotient of two real numbers when $b \neq 0$, but as a symbol $a / 0$ when $b=0$. Using the notation (3.3), the hypothesis (3.1) in the theorem given above may be omitted.

Theorem 3.2. Suppose a function $f$ defined in $\mathbf{R}^{n}$ is differentiable and its gradient is quasidifferentiable. Then for any $x \in \mathbf{R}^{n}, \alpha \neq 0, \alpha \in \mathbf{R}^{n}$ and $\lambda>0$, one has the following second-order expansion (Taylor's expansion of second order)

$$
\begin{gathered}
f(x+\lambda \alpha) \in f(x)+\lambda f^{\prime}(x ; \alpha)+\frac{\lambda^{2}}{2} \alpha^{T}\left[\partial^{2} f(x+\xi \alpha)\right. \\
\left.+\partial^{2} f(x+\xi \alpha)\right] \alpha,
\end{gathered}
$$

where $\xi \in(0, \lambda)$, or an alternative expression

$$
f(x+\lambda \alpha)=f(x)+\lambda \rho^{\prime}(x ; \alpha)+\frac{\lambda^{2}}{2} \alpha^{T}(V+W) \alpha
$$

for some $V$ and $W$, where

$$
V \in \underline{\theta}^{2} f(x+\xi d)
$$

and

$$
W \in \bar{\theta}^{2} f(x+\xi \alpha) . \quad \xi \in(0, \lambda) .
$$

Proof. Making auxiliary functions

$$
\varphi(t)=f(x+\lambda \alpha)-f(x+t \alpha)-(\lambda-t) f^{\prime}\left(x+t \alpha^{\prime} ; \alpha^{\prime}\right)
$$

and

$$
\psi(t)=(\lambda-t)^{2}
$$

We have from the rules of quasidifferential calculus [3] that

$$
D \varphi(t)=-D_{t} f(x+t \alpha)-f^{\prime}(x+t \alpha ; \alpha) D_{t}(\lambda-t)
$$




$$
-(\lambda-t) D_{t} f^{\prime}(x+t \alpha ; \alpha)
$$

and

$$
D \psi(t)=2(\lambda-t) D(\lambda-t)
$$

where $D_{t} f$ means a quasidifferential of $f$ with respect to $t$. We now evaluate every term on the right-hand-side of (3.6). For the first term it can be expressed as

$$
D_{t} f(x+t \alpha)=[\langle\partial f(x+t \alpha), \alpha\rangle,\langle\bar{\partial} f(x+t \alpha), \alpha\rangle]
$$

Since $f$ is differentiable in $R^{n}$, one has

$$
D_{t} f(x+t d)=\left[f^{\prime}(x+t \alpha ; \alpha), 0\right]
$$

The second term is

$$
f^{\prime}\left(x+t \alpha ; \alpha^{\prime}\right)[0,1]
$$

In the third term we have from the Proposition 2.5. that

$$
D_{t} f^{\prime}(x+t \alpha ; \alpha)=\left[\alpha^{T} \diamond_{1} D^{2} f(x+t \alpha) \alpha^{T}, \alpha^{T} \diamond_{2} D^{2} f(x+t \alpha) \alpha^{T}\right] .
$$

From the Cauchy's Mean Value Theorem given above it follows that there exists a $\xi \in(0, \lambda)$ such that

$$
\frac{\varphi(\lambda)-\varphi(0)}{\psi(\lambda)-\psi(0)} \in \frac{\langle\theta \varphi(\xi)+\bar{\partial} \varphi(\xi), \lambda\rangle}{\underline{\theta} \psi(\xi)+\bar{\partial} \psi(\xi), \lambda\rangle}
$$

where

$$
\varphi(\lambda)=0, \varphi(0)=f(x+\lambda \alpha)-f(x)-\lambda f^{\prime}(x ; \alpha), \psi(\lambda)=0, \psi(0)=\lambda^{2} .
$$

From (3.6), (3.8), (3.9), (3.10) (3.11) and (3.7) one has

$$
\begin{gathered}
\partial \varphi(\xi)+\bar{\partial} \varphi(\xi)=-\alpha^{T}(\lambda-\xi)\left[\partial^{2} \rho(x+\xi \alpha)+\bar{\theta}^{2} \rho(x+\xi \alpha)\right] \\
\partial \psi(\xi)+\bar{\partial} \psi(\xi)=-2(\lambda-\xi) .
\end{gathered}
$$

It follows from substituting the expressions above into (3.12) that

$$
\frac{f(x+\lambda \alpha)-f(x)-\lambda f^{\prime}(x ; \alpha)}{\lambda^{2}} \in \frac{(\lambda-\xi) \alpha^{T}\left[\partial^{2} \rho(x+\xi \alpha)+\bar{\theta} f(x+\xi \alpha)\right] d}{2(\lambda-\xi)},
$$

and hence

$$
f(x+\lambda \alpha) \in f(x)+\lambda f^{\prime}(x ; \alpha)+\frac{\lambda^{2}}{2} \alpha^{T}\left[\theta^{2} f(x+\xi \alpha)\right.
$$




$$
\left.+\bar{\partial}^{2} f(x+\xi d)\right] d,
$$

where $\xi \in(0, \lambda)$.

Corollary 3.3. Under the assumptions given in the above theorem, for any $\lambda>0$ small enough there exists a $v \in(0, \lambda)$ such that

$$
f(x+\lambda \alpha)=f(x)+\lambda f^{\prime}(x ; \alpha)+\lambda \vartheta f^{\prime \prime}(x ; \alpha, \alpha)+o\left(\lambda^{2}\right) .
$$

Proof. From the above theorem and the Mean Value Theorem we have

$$
f^{\prime}(x+\vartheta \alpha ; \alpha)-f^{\prime}(x ; \alpha)=\frac{\lambda}{2} \alpha^{T}(V+W) \alpha
$$

where $\vartheta \in(0, \lambda)$ and

$$
V \in \underline{\partial}^{2} \rho(x+\xi \alpha), W \in \bar{\partial}^{2} \rho(x+\xi \alpha), \xi \in(0, \lambda) .
$$

Taking limits to (14) $/ \lambda$ as $\lambda+0$, we obtain

$$
\lim _{\lambda+0} \frac{f^{\prime}(x+\lambda \alpha ; \alpha)-f^{\prime}(x ; \alpha)}{\vartheta}=\lim _{\lambda \downarrow 0} \alpha^{T}\left[\frac{\lambda}{2 \vartheta}(V+W)\right]^{\alpha}
$$

Since $f^{\prime \prime}\left(x ; \alpha^{\prime}, \alpha^{\prime}\right)$ exists and $\vartheta \rightarrow 0^{+}$as $\lambda \downarrow 0$, the following equalities,

$$
\begin{aligned}
\lim _{\lambda \downarrow 0} \frac{1}{\vartheta} & {\left[f^{\prime}(x+\vartheta d ; d)-f^{\prime}(x ; d)\right] } \\
& =\lim _{\lambda \perp 0} \frac{1}{\lambda}\left[f^{\prime}(x+\lambda d ; d)-f^{\prime}(x ; d)\right] \\
& =f^{\prime \prime}(x ; d, d) .
\end{aligned}
$$

hold. Thus

$$
\frac{\lambda^{2}}{2} d^{T}(V+W) d^{T}=\lambda \vartheta f^{\prime \prime}(x ; d, d)+\lambda \vartheta \varepsilon
$$

where $\varepsilon \rightarrow 0$ as $\lambda \rightarrow 0,0<\vartheta<\lambda$. The (3.13) can be deduced immediately from (15).

EIUO CONDITION (the condition of exchangeability of intersection and union operations) is defined as follows:

Let $C$ be a family of nonempty compact convex set and $A$ be a nonempty compact convex set. We say that $C$ and $A$ satisfy the EIUO condition if the following condition holds 


$$
\bigcup_{\alpha \in A Z \in C}[Z-\alpha]=\bigcap_{Z \in C a \in A} \cup[Z-\alpha]
$$

Lemma 3.4. If for some $\left[U_{1}, U_{2}\right] \in D^{2} f(x)$ the family $\left\{\underline{\partial}^{2} f(x)+\bar{\partial}^{2} f(x)\right\}$ and set $U_{2}$ satisfy the EIUO condition, and also $\left\{\bar{\partial}^{2} f(x)-\bar{\partial}^{2} f(x)\right\}$ and $U_{1}$ satisfy the EIUO condition, where $D^{2} f(x)$ denotes the class of quasidifferentials of $f$ at $x$, then

$$
\left[\bigcap_{\left[V_{1}, V_{2}\right] \in D^{\varepsilon_{f}(x)}}\left(V_{1}+V_{2}\right), \quad \bigcap_{\left[V_{1}, V_{2}\right] \in D^{\varepsilon_{f}\langle x\rangle}}\left(V_{2}-V_{2}\right)\right] \in D^{2} f(x)
$$

Remark. If $\left[V_{1}, V_{2}\right] \in D^{2} f(x)$, then $\left[V_{1}+V_{2}, V_{2}-V_{2}\right] \in D^{2} f(x)$.

Proof of the Lemma. It is clear that

$$
\bigcap_{\left.1, V_{\varepsilon}\right] \in D^{\mathbb{E}} f(x)}\left[U_{1}-\left(V_{1}-V_{2}\right)\right]
$$

is a nonempty compact convex set as inasmuch $0 \in V_{2}-V_{2}$ and

$$
U_{1} \subset \bigcap_{\left[V_{1}, V_{2}\right] \in D^{\mathbb{R}_{f}}(x)}\left[U_{1}-\left(V_{2}-V_{2}\right)\right] .
$$

Since $\left[U_{1}, U_{2}\right],\left[V_{1}, V_{2}\right] \in D^{2} f(x)$ and the foregoing remark, we have

$$
\bigcap_{\left[V_{1}, V_{g}\right] \in D^{\mathbb{E}} f(x)}\left[U_{1}-\left(V_{2}-V_{2}\right)\right]=\bigcap_{\left[V_{1}, V_{\varepsilon}\right] \in D^{\mathbb{Z}} f\langle x\rangle}\left[\left(V_{1}+V_{2}\right)-U_{2}\right] .
$$

From this the right hand side of the above expression is a nonempty compact convex set. According to the EIUO condition

$$
\left\{\underline{\theta}^{2} f(x)+\bar{\partial}^{2} f(x)\right\}
$$

and $U_{2}$ satisfy the equality,

$$
\bigcap_{\left[V_{1}, V_{2}\right] \in D^{\mathbb{R}_{f}}(x)}\left(V_{1}+V_{2}\right)-U_{2}=\bigcap_{\left[V_{1}, V_{2}\right] \in D^{2_{f}} f(x)}\left[\left(V_{1}+V_{2}\right)-U_{2}\right] .
$$

since

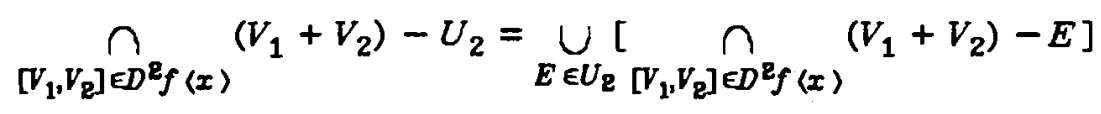

$$
\begin{aligned}
& =U \cap\left(V_{1}+V_{2}-E\right)
\end{aligned}
$$

and 


$$
\bigcap_{\left[V_{1}, V_{2}\right] \in D^{2_{f}}(x)}\left(V_{1}+V_{2}-U_{2}\right)=\underset{\left[V_{1}, V_{2}\right] \in D^{\mathbf{2}_{f}}(x) E \in U_{2}}{\bigcup}\left(V_{1}+V_{2}-E\right)
$$

Similarly, one has

$$
U_{1}-\bigcap_{\left[V_{1}, V_{2}\right] \in D f(x)}\left(V_{1}-V_{2}\right)=\bigcap_{\left[V_{1}, V_{2}\right] \in D^{2} f(x)}\left[U_{1}-\left(V_{1}-V_{2}\right)\right] .
$$

It can be asserted from (17), (18) and (19) that two intersections

$$
\bigcap_{\left[V_{1}, V_{R}\right] \in D^{2} f(x)}\left(V_{1}+V_{2}\right)
$$

and

$$
\bigcap_{\left[V_{1}, V_{2}\right] \in D^{R_{f}(x)}}\left(V_{2}-V_{2}\right)
$$

are nonempty compact convex sets, and

$$
U_{1}-\bigcap_{\left[V_{1}, V_{2}\right] \in D^{2} f(x)}\left(V_{2}-V_{2}\right)=\bigcap_{\left[V_{1}, V_{2}\right] \in D^{2} f(x)}\left(V_{1}+V_{2}\right)-U_{2}
$$

Hence the lemma is true.

By $\underline{D}^{2} f(x)$ and $\bar{D}^{2} f(x)$ we denote the

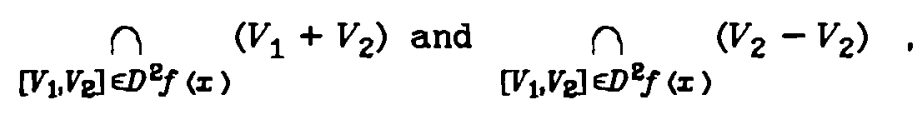

respectively, when the conditions in the foregoing lemma are satisfied.

Theorem 3.5. Suppose conditions indicated in the foregoing lemma are satisfied, and assume furthermore that mappings $\underline{D}^{2} f(x)$, defined by

$$
x \rightarrow \underline{D}^{2} f(x)=\bigcap_{\left[V_{1}, V_{2}\right] \in D^{2} f(x)}\left(V_{1}+V_{2}\right)
$$

and $D^{2} f(x)$, defined by

$$
x \rightarrow \bar{D}^{2} \rho(x)=\bigcap_{\left[V_{1}, V_{R} \in D^{2} f(x)\right.}\left(V_{2}-V_{2}\right)
$$

are upper semi-continuous in a neighborhood in a direction $\alpha \neq 0$. Then for any $\varepsilon>0$ there exists a $\bar{\lambda}$ small enough such that one has a Taylor's expansion of second-order as follows:

$$
f(x+\lambda \alpha) \in f(x)+\lambda f^{\prime}(x ; \alpha)+\frac{\lambda^{2}}{2} \alpha^{T}\left(D^{2} f(x)+\bar{D}^{2} f(x)\right) \alpha
$$




$$
+\lambda^{2} \varepsilon \alpha^{T} B
$$

where $\lambda \in[0, \bar{\lambda}], \beta_{1}^{2 n}(0)$ is a unit ball in $\mathbf{R}^{2 n}$.

Proof. The proof of this theorem can be done in terms of Theorem 3.2, Lemma 3.4 and upper semi-continuity of $\underline{D}^{2} f(x)$ and $\bar{D}^{2} f(x)$ in the sense of the above lemma.

\section{SECOND-ORDER QUASIDIFFERENTIALS OF A COMPOSITION}

We will deduce a formula for second-order quasidifferentials of a composite function in this section.

Definition 4.1. ([4]. [8]). Let $X$ and $Y$ be Banach spaces, and $H$ be a mapping: $\Omega \rightarrow Y$ where $\Omega$ is an open set in $X$. The mapping $H$ is called uniformly directionally differentiable at this point and if for any $\alpha \in X$ and $\varepsilon>0$ there exist numbers $\delta>0$ and $\alpha_{0}>0$ such that the inequality

$$
\left\|H(x+\alpha v)-H(x)-a H^{\prime}(x ; v)\right\|<\alpha \varepsilon
$$

holds for all $v \in B_{\delta}^{\circ}(\alpha), a \in\left[0, \alpha_{0}\right]$, where

$$
B_{\delta}^{\circ}(\alpha)=\{v \mid\|\alpha-v\|<\delta\} .
$$

$H$ is referred to as uniformly quasidifferentiable at $x$ if $H$ is uniformly directionally differentiable and quasidifferentiable at $\boldsymbol{x}$.

Lemma 4.2 [4]. Let $X, Y$ and $Z$ be Banach spaces, and $\Omega_{x}, \Omega_{y}$ and $\Omega_{z}$ be open sets, respectively, in $X, Y$ and $Z$. A mapping $H: X \rightarrow Y$ is directionally differentiable at a point $X \in \mathrm{n}_{X}$, and a mapping $G: Y \rightarrow Z$ is uniformly directionally differentiable at $H(x)$. Then the mapping $F=G \cdot H$ is directionally differentiable at $x$ and

$$
\begin{aligned}
F^{\prime}(x ; \alpha) & =G^{\prime}\left(H(x) ; H^{\prime}(x ; \alpha)\right) \\
& =\left(G^{\prime}{ }_{H(x)} \cdot H^{\prime}{ }_{x}\right)(\alpha) \\
& \forall \alpha \in X .
\end{aligned}
$$

Theorem 4.3. Let $f: \mathbf{R}^{n} \rightarrow \mathbf{R}^{m}$ where $f=\left(f_{1}, \ldots, f_{m}\right)^{T}$ and $\varphi: \mathbf{R}^{m} \rightarrow \mathbf{R}^{n}$. Suppose $f$ and $\varphi$ are continuously differentiable in some neighborhoods, respectively, of $x$ and $f(x)$, and assume furthermore that the gradient of $x, \nabla f(x)$, is quasidifferentiable in that neighborhood of $x$, and th gradient of $\varphi$ is uniformly quasidif- 
ferentiable in that neighborhood of $f(x)$. Then $(\varphi \cdot f)$ is twice quasidifferentiable at $\boldsymbol{x}$ and the following formulas hold:

$$
(\varphi \cdot f)^{\prime \prime}\left(x ; d_{1}, d_{2}\right)=\left(\varphi_{f(x)}^{\prime} \cdot f^{\prime \prime}{ }_{x}+\varphi^{\prime \prime}{ }_{f(x)} \cdot\left[f^{\prime}{ }_{x}\right]\right)\left(d_{1}, d_{2}\right),
$$

where

$$
\begin{aligned}
{\left[f^{\prime}{ }_{x}\right]\left(\alpha_{1}, \alpha_{2}\right): } & =\left(f^{\prime}{ }_{x}\left(\alpha_{1}\right), f^{\prime}{ }_{x}\left(\alpha_{2}\right)\right) \\
& =\left(f^{\prime}\left(x ; \alpha_{1}\right), f^{\prime}\left(x ; \alpha_{2}\right)\right),
\end{aligned}
$$

and

$$
\begin{aligned}
D^{2}(\varphi \cdot f)(x) & =\nabla_{f}^{T} \varphi(f(x)) \cdot D^{2} f(x) \\
& +J^{T} f(x) \cdot D_{f}^{2} \varphi(f(x)) J f(x) .
\end{aligned}
$$

where

$$
\begin{gathered}
D^{2} f:=\left[\begin{array}{c}
D^{2} f_{1} \\
\vdots \\
D^{2} f_{m}
\end{array}\right], \\
J^{T} f(x) D_{f}^{2} \varphi(f(x)) J f(x)=\left[J^{T} f(x) \cdot \underline{\theta}_{f}^{2} \varphi(f(x)) J f(x),\right. \\
\left.J^{T} f(x)\left(\bar{\partial}_{f}^{2} \varphi(f(x))\right) J f(x)\right] .
\end{gathered}
$$

Proof. Since

$$
(\varphi \cdot f)^{\prime}\left(x ; d_{1}\right)=\left\langle J^{T} f(x) \cdot \nabla_{f} \varphi(f(x)), \alpha_{1}\right\rangle .
$$

$\varphi \cdot f$ is twice directionally differentiable at $x$. We now calculate the second-order directional derivative at $x$. To begin with, it follows from the definition of directional derivative that

$$
\begin{aligned}
(\varphi \cdot f)^{\prime \prime} & \left(x ; \alpha_{1}, \alpha_{2}\right)=\lim _{\lambda \downarrow 0} \frac{1}{\lambda}\left[\left\langle\nabla(\varphi \cdot f)\left(x+\lambda \alpha_{2}\right), \alpha_{1}\right\rangle\right. \\
& \left.-\left\langle\nabla(\varphi \cdot f)(x), \alpha_{1}\right\rangle\right] \\
& =\lim _{\lambda \downarrow 0} \frac{1}{\lambda}\left\langle\left[J f\left(x+\lambda \alpha_{2}\right)-J f(x)\right]^{T} \nabla_{f} \varphi\left(f\left(x+\lambda \alpha_{2}\right), \alpha_{1}\right\rangle\right. \\
& +\lim _{\lambda \downarrow 0} \frac{1}{\lambda}\left\langle J^{T} f(x)\left[\nabla_{f} \varphi\left(f\left(x+\lambda \alpha_{2}\right)-\nabla_{f} \varphi(f(x))\right], \alpha_{1}\right\rangle .\right.
\end{aligned}
$$

The first limit in (4.4) equals 


$$
\begin{aligned}
& \lim _{\lambda+0_{i}} \sum_{i=1}^{m}\left[\left[\frac{\partial \varphi}{\partial f_{1}}\left(\rho\left(x+\lambda \alpha_{2}\right)\right)\right]\left[\frac{\left\langle\nabla f_{1}\left(x+\lambda \alpha_{2}\right)-\nabla f_{1}(x), \alpha_{1}\right\rangle}{\lambda}\right]\right] \\
& =\sum_{i=1}^{m} \frac{\partial \varphi(f(x))}{\partial f_{i}} \lim _{\lambda+0} \frac{1}{\lambda}\left[f_{i}^{\prime}\left(x+\lambda \alpha_{2} ; d_{1}\right)-f^{\prime}{ }_{i}(x ; \alpha)\right] \\
& =\sum_{i=1}^{m} \frac{\partial \varphi(f(x))}{\partial f_{i}} f^{\prime \prime}{ }_{i}\left(x ; \alpha_{1}, \alpha_{2}\right) \\
& =\left(\varphi_{f(x)}^{\prime} \cdot f^{\prime \prime}{ }_{x}\right)\left(d_{1}, d_{2}\right) .
\end{aligned}
$$

We have obtained the first part of the right hand side of the expression (4.4).

The second limit on the right hand side of (4.4) can be developed as follows. It can be rewritten in the following form:

$$
\begin{aligned}
& \lim _{\lambda \rightarrow 0} \frac{1}{\lambda}\left[\varphi_{f}^{\prime}\left(f\left(x+\lambda \alpha_{2}\right) ; J f(x) \alpha_{1}\right)-\varphi_{f}^{\prime}\left(f(x) ; J f(x) \alpha_{1}\right)\right] \\
& =\lim _{\lambda \downarrow 0} \frac{1}{\lambda}\left\langle\left(\left(\nabla_{f} \varphi\right) \cdot f\right)\left(x+\lambda \alpha_{2}\right)-\left(\left(\nabla_{f} \varphi\right) \cdot f\right)(x), J f(x) \alpha_{1}\right\rangle .
\end{aligned}
$$

From the lemma 4.2 the above expression becomes

$$
\begin{aligned}
& \left\langle\left(\left(\nabla_{f} \varphi\right)_{f(x)}^{\prime} \cdot f^{\prime}{ }_{x}\right)\left(\alpha_{2}\right), J f(x) \alpha_{1}\right\rangle \\
& =\left\langle\left(\nabla_{f} \varphi\right)^{\prime}\left(f(x) ; J f(x) \alpha_{2}\right), J f(x) d_{1}\right\rangle .
\end{aligned}
$$

Let $v=J f(x) d_{1}$ and $u=J f(x) d_{2}$. then the expression turns to

$$
\begin{aligned}
<\left(\nabla_{f} \varphi\right)^{\prime}(f(x) ; u), v> & =\left(\varphi_{f}^{\prime}(x)(v)\right)_{f(x)}(u) \\
& =\varphi^{\prime \prime} f(f(x) ; v, u) \\
& =\varphi^{\prime \prime}{ }_{f}\left(f(x) ; J f(x) d_{1}, J f(x) d_{2}\right) \\
& =\left(\varphi^{\prime \prime} f(x) \cdot\left[f^{\prime}\right]_{x}\right)\left(d_{1}, d_{2}\right) .
\end{aligned}
$$

This is what we want for the second limit in (4.4).

The demonstration of (4.3) will be done below. Since

$$
\begin{aligned}
(\varphi \cdot f)^{\prime \prime}\left(x ; \alpha_{1}, \alpha_{2}\right) & =\varphi^{\prime}\left(f(x) ; f^{\prime \prime}\left(x ; \alpha_{1}, \alpha_{2}\right)\right) \\
& +\varphi^{\prime \prime}\left(f(x) ; J f(x) \alpha_{1}, J f(x) \alpha_{2}\right)
\end{aligned}
$$

one has to calculate the two terms on the right hand side of (4.7), 
$\varphi^{\prime}\left(f(x) ; f^{\prime \prime}\left(x ; \alpha_{1}, \alpha_{2}\right)\right)$ and $\varphi^{\prime \prime}\left(f(x) ; J f(x) \alpha_{1}, J f(x) \alpha_{2}\right)$, respectively. Let

$$
\lambda_{1}=\frac{\partial \varphi}{\partial f_{1}}(f(x))
$$

We have

$$
\begin{aligned}
\varphi^{\prime}\left(f(x) ; f^{\prime \prime}\left(x ; \alpha_{1}, \alpha_{2}\right)\right) & =\left\langle\nabla_{f} \varphi(f(x)), f^{\prime \prime}\left(x ; \alpha_{1}, \alpha_{2}\right)\right\rangle \\
& =\sum_{i=\alpha}^{m} \frac{\partial \varphi}{\partial f_{i}}(f(x)) f^{\prime \prime}\left(x ; \alpha_{1}, \alpha_{2}\right) \\
& =\sum_{i=1}^{m}\left(\lambda_{i} f_{i}\right)^{\prime \prime}\left(x ; \alpha_{1}, \alpha_{2}\right) \\
& =\left(\sum_{i=1}^{m} \lambda_{i} f_{i}\right)^{\prime \prime}\left(x ; \alpha_{1}, \alpha_{2}\right) .
\end{aligned}
$$

We now calculate the second term on the right hand side of (4.7). Since

$$
\begin{aligned}
& \varphi^{\prime \prime}\left(f(x) ; J f(x) \alpha_{1}, J f(x) \alpha_{2}\right)= \\
& =\lim _{\lambda \downarrow 0} \frac{1}{\lambda}\left\langle\nabla_{f} \varphi\left(f(x)+\lambda J f(x) \alpha_{2}\right)-\nabla_{f} \varphi(f(x)), J f(x) \alpha_{1}\right\rangle \\
& =\lim _{\lambda \downarrow 0} \frac{1}{\lambda}\left\langle J^{T} f(x) \cdot \nabla_{f} \varphi\left(f(x)+\lambda J f(x) \alpha_{2}\right)-J^{T} f(x) \cdot \nabla_{f} \varphi(f(x)), \alpha_{1}\right\rangle .
\end{aligned}
$$

one has from the hypotheses in this theorem that

$$
\varphi^{\prime \prime}\left(f(x) ; J f(x) \alpha_{1}, J f(x) \alpha_{2}\right)=\left\langle\left(J^{T} f(x) \cdot \nabla_{f} \varphi\right)^{\prime}\left(f(x) ; J f(x) \alpha_{2}, \alpha_{1}\right\rangle .\right.
$$

Since

$$
\begin{aligned}
& \left(J^{T} f(x) \cdot \nabla_{f} \varphi\right)^{\prime}\left(f(x) ; J f(x) d_{2}\right) \\
& =\max _{V \varepsilon_{d}\left\langle J^{T} f(x) \cdot \nabla_{f} \varphi(f(x))\right)} V \cdot J f(x) \alpha_{2}+\min _{W \in \bar{J}_{D}\left\langle J^{T} f(x) \cdot \nabla_{f} \varphi(f(x))\right)} W \cdot J f(x) \alpha_{2} \\
& =\max _{V \in J^{T} f(x) J_{D} \nabla_{f} \varphi(f(x)) J f(x)} V \alpha_{2}+\min _{W \in J^{T} f(x) V_{D} \nabla_{f} \varphi(f(x)) J f(x)} W \cdot \alpha_{2} \\
& =\max _{V \in J^{T} f(x) \theta_{f}^{2} \varphi(f(x)) J f(x)} V \alpha_{2}+\operatorname{W\in J}_{J^{T} f(x)-\min _{\varphi}(f(x)) J f(x)} W \alpha_{2} .
\end{aligned}
$$

one has from the proof of Theorem 2.4 that letting

$$
\psi^{\prime \prime}\left(x ; \alpha_{1}, \alpha_{2}\right)=\varphi^{\prime \prime}\left(f(x) ; J f(x) \alpha_{1}, J f(x) \alpha_{2}\right)
$$


we have

$$
D^{2} \psi(x)=J^{T} f(x) \cdot D_{f}^{2} \varphi(f(x)) \cdot J f(x)
$$

Combining (4.8) and (4.9), we obtain (4.3). The proof has been completed.

\section{NECESSARY CONDITIONS FOR UNCONSTRAINED PROBLEMS}

Theorem 5.1. Under the assumptions given in the Theorem 3.9, suppose $x$ is a local minimum point to minimize $f(x), x \in \mathbf{R}^{n}$. Then for every nonzero $d \in \mathbf{R}^{n}$ there exist $V \in \partial^{2} f(x)$ and $W \in \bar{\partial}^{2} f(x)$ such that

$$
d^{T}(V+W) d \geq 0 \text {. }
$$

or

$$
d^{T} H d \geq 0
$$

where $H \in \partial^{2} f(x)+\bar{\partial}^{2} f(x)$.

Proof. Suppose for any $V, V^{\prime} \in \underline{\partial}^{2} f(x)$ and $W, W^{\prime} \in \bar{\theta}^{2} f(x)$.

$$
d^{+T}\left(V+W^{\prime}\right) d-d^{-T}\left(W+V^{\prime}\right) d<0 .
$$

Then exists a nonzero $d \in \mathbf{R}^{n}$ such that

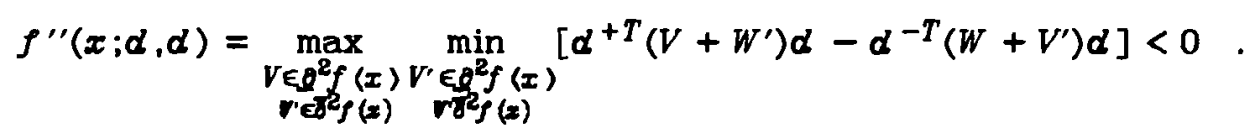

Since $f$ is differentiable, the gradient of $f$ at $x$ equals zero, i.e., $\nabla f(x)=0$. In other words $f^{\prime}(x ; d)=0$. According to (3.13) we have

$$
f(x+\lambda \alpha)-f(x)=\lambda \vartheta \rho^{\prime \prime}(x ; \alpha, \alpha)+o(\lambda),
$$

where $\vartheta \in(0, \lambda)$. Thus for $\lambda>0$ small enough one has

$$
f(x+\lambda d)-f(x)<0
$$

The (5.2) shows that $x$ is not a local minimum point. This leads to that there exist $V \in \partial^{2} f(x)$ and $W \in \bar{\partial}^{2} f(x)$ such that

$$
\begin{gathered}
\alpha^{+T}\left(V+W^{\prime}\right) d-\alpha^{-T}\left(W+V^{\prime}\right) \alpha \geq 0 \\
\forall\left(V^{\prime}, W^{\prime}\right) \in D^{2} f(x)=\left[\partial^{2} f(x), \bar{\theta}^{2} f(x)\right]
\end{gathered}
$$


holds. Taking $V^{\prime}=V$ and $W^{\prime}=W$, we have

$$
d^{T}(V+W) d \geq 0 .
$$

The proof is completed.

Theorem 5.2. Under the assumptions given in the Theorem 3.2, suppose $x$ is a local minimum point to minimize $f(x), x \in \mathbb{R}^{n}$. Then for each pair of $V \in \underline{\partial}^{2} f(x)$ and $W \in \bar{\partial}^{2} f(x)$ there exist vectors $g_{1}, g_{2} \in B_{1}(0)$ such that

$$
g_{2}^{T} V-g_{1}^{T} W \in g_{1}^{T} \partial^{2} f(x)-g_{2}^{T} \partial^{2} f(x)
$$

where

$$
\begin{gathered}
g_{1}=\sum \lambda_{i} \alpha_{i}+T, g_{2}=\sum \lambda_{i} \alpha_{i}-T \\
\alpha_{i} \in b \alpha B_{1}(0), i=1, \ldots, m, \\
\lambda_{i} \geq 0, i=1, \ldots, m, \\
\sum \lambda_{i}=1 .
\end{gathered}
$$

Remark. The expression (5.3) can be rewritten as

$$
\begin{aligned}
\left(\sum \lambda_{i} \alpha_{i}{ }^{-T} V-\right. & \left(\sum \lambda_{i} \alpha_{i}^{+T}\right) W \in \\
& \left(\sum \lambda_{i} \alpha_{i}+T\right) \underline{\partial}^{2} f(x)-\left(\sum \lambda_{i} \alpha_{i}{ }^{-T}\right) \bar{\partial}^{2} f(x) .
\end{aligned}
$$

or

$$
-\sum \lambda_{i} \alpha_{i}^{T} \diamond_{2}(V, W) \in \sum \lambda_{i} \alpha^{T} \diamond_{1} D^{2} f(x)
$$

Proof. From the proof of the above theorem one has that for any $d \in \mathbf{R}^{n}$, the following holds

$$
\begin{aligned}
& f^{\prime \prime}(x ; \alpha, \alpha)=f^{\prime \prime}(x ; \alpha) \\
& =\min _{\substack{V \in g^{2} f(x) \\
V \in J_{f}^{2}(x)}} \max _{\substack{V^{\prime} \in g^{2} f(x) \\
V^{\prime} \in \mathcal{U}^{2} f(x)}}\left[\alpha^{+T}\left(V^{\prime}+W\right)-\alpha^{-T}\left(V+W^{\prime}\right)\right] d^{T}
\end{aligned}
$$

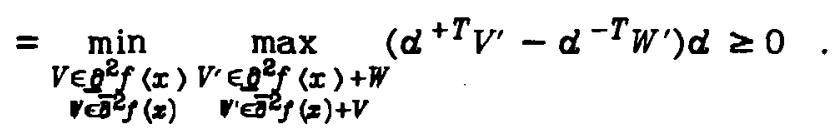

It follows from this that

$$
\min _{\| d \mid=1} \min _{\substack{V \in g^{2} f(x) \\ v \in d^{2} f(x)}} \max _{V^{\prime} g^{2} f(x)}\left(\alpha^{+T} V^{\prime}-\alpha^{-T} W^{\prime}\right) \alpha \geq 0 .
$$


A closed convex set depending on $V$ and $W$ can be constructed as follows:

$$
\begin{aligned}
Q(V, W):= & \operatorname{coQ} Q^{\prime}(V, W) \\
= & \operatorname{co}\left\{u=\alpha^{+}+V^{\prime}-\alpha^{-T} W^{\prime} \mid V^{\prime} \in \underline{\partial}^{2} f(x)+W,\right. \\
& \left.W^{\prime} \in \bar{\partial}^{2} f(x)+V,\|\alpha\|=1\right\} .
\end{aligned}
$$

Hence

$$
\min _{\|d\|=1} \min _{\substack{V \in Q^{2} f(x) \\ V \in \Theta^{2} f(x)}} \max _{u \in Q\langle V, W)}\left\langle u^{T}, d\right\rangle \geq 0 .
$$

i.e.,

$$
\min _{\|d\|=1} \max _{u \in Q(V, W)}\left\langle u^{T}, d\right\rangle \geq 0
$$

for each pair of $V \in \partial^{2} f(x)$ and $W \in \bar{\partial}^{2} f(x)$. According to [6, Lemma 1] or [3], we have the following assertion:

$$
O \in Q(V, W)
$$

The (5.6) shows that there exist $d_{i} \in b \alpha B_{1}(0), i=1, \ldots, m$, $\lambda_{i} \geq 0, i=1, \ldots, m, \sum \lambda_{i}=1$ such that

$$
0 \in \sum \lambda_{i}\left(\alpha_{i}^{+T}\left(\partial^{2} f(x)+W\right)-\alpha^{-T}\left(\bar{\partial}^{2} f(x)+V\right)\right) .
$$

i.e.,

$$
\begin{aligned}
0 \in\left(\sum \lambda_{i} \alpha_{i}+T\right) W & -\left(\sum \lambda_{i} \alpha_{i}^{-T}\right) V+\sum\left(\lambda_{i} \alpha_{i}+T^{2} f(x)\right) \\
& -\sum\left(\lambda_{i} \alpha_{i}{ }^{-T} \bar{\partial}^{2} f(x)\right) .
\end{aligned}
$$

Since $\lambda_{i} \alpha_{i}{ }^{+T}$ and $\lambda_{i} \alpha_{i}{ }^{-T}$ are nonnegative, one has

$$
\begin{gathered}
\left(\sum \lambda_{i} \alpha_{i}{ }^{-T}\right) V-\left(\sum \lambda_{i} \alpha_{i}^{+T}\right) W \in\left(\sum \lambda_{i} \alpha_{i}{ }^{+T}\right) \underline{\partial}^{2} f(x) \\
-\left(\sum \lambda_{i} \alpha_{i}{ }^{-T}\right) \bar{\partial}^{2} f(x) .
\end{gathered}
$$

Letting

$$
g_{1}=\sum \lambda_{i} \alpha_{t}^{+}, g_{2}=\sum \lambda_{i} \alpha_{i}^{-}
$$

we obtain the form (5.3). The proof is completed. 


\section{References}

[1] Clarke, F.H. (1976). On the inverse function theorem. Pacific Journal of Mathematics, 64, pp. 97-102.

[2] Clarke, F.H. (1983). Optimization and nonsmooth analysis. Wiley - Interscience, New York.

[3] Demyanov, V.F. and Vasiliev, L.V. (1981). Nondifferentiable optimization. Nauka, Moscow.

[4] Demyanov, V.F. and Rubinov, A.M. On quasidifferentiable mappings. Mathematische Operationsforschung und Statistik, Series Optimization 14, pp. 3-21.

[5] Hiriart-Urruty, J.-B., Strodiot, J.-J. and Nguyen, V.H. (1984). Generalized Hessian matrix and second-order optimality conditions for problems with $C^{1.1}$ data. Appl. Math. Optim., 11, pp. 43-56.

[6] Polyakova, L.N. (1981). Necessary conditions for an extremum of quasidifferentiable functions. Vestnik, Leningrad Univ. Math., Vol. 13, pp. 241-247.

[7] Xia, Z.Q. (1984). On mean value theorems in quasidifferential calculus. Abstracts of the IIASA Workshop on Nondifferentiable Optimization: Motivations and Applications, Sopron, Hungary, 17-22 September 1984, IIASA, Laxenburg, Austria.

[8] Demyanov, V.F. and Rubinov, A.M. (1968). Approximate Methods for the solution of extremal problems. Leningrad State University Press. 